\title{
Basic Properties and Characterizations of Incremental Stability Prioritizing Flow Time for a Class of Hybrid Systems
}

\author{
Yuchun $\mathrm{Li}^{1, *}$, Sean Phillips ${ }^{1}$, Ricardo G. Sanfelice ${ }^{1}$
}

\begin{abstract}
This paper introduces incremental stability notions for a class of hybrid dynamical systems given in terms of differential equations and difference equations with state constraints. The specific class of hybrid systems considered are those that do not have consecutive jumps nor Zeno behavior. The notion of incremental asymptotic stability is used to describe the behavior of the distance between every pair of solutions to the system having stable behavior (incremental stability) and approaching zero asymptotically (incremental attractivity). A version of this notion that is uniform (in hybrid time) with respect to initial conditions is also introduced. These notions prioritize flow time and are illustrated in examples. Basic properties of the class of systems are considered and those implied by the new notions are revealed. An equivalence characterization of the uniform notion is provided in terms of a $\mathcal{K} \mathcal{L}$-function. Moreover, sufficient and necessary conditions under which asymptotic stability implies the new incremental notions are provided. We consider the case when the original hybrid system has an asymptotically stable compact set and also the case when an auxiliary hybrid system, which has twice the dimension of the original system, has a diagonal-like set asymptotically stable.
\end{abstract}

Keywords: Hybrid systems, incremental stability, asymptotic stability.

\footnotetext{
${ }^{1}$ Y. Li, S. Phillips, and R. G. Sanfelice are with the Department of Computer Engineering, University of California, Santa Cruz, CA 95064. Tel. 001 831-459-1016. Email: yuchunli, seaphill, ricardo@ucsc.edu.
} 


\section{Introduction}

\subsection{Background}

Incremental stability is the notion that the distance between any two solutions to the system has stable behavior and converges to zero. Specifically, in its uniform using a uniform version of such notion ${ }^{2}$, a continuous-time system $\dot{x}=f(x)$ is said to be incrementally stable if there exists a class- $\mathcal{K} \mathcal{L}$ function $\beta$ (a formal definition is provided in Section 1.3) such that every pair of solutions $t \mapsto \phi_{1}(t)$ and $t \mapsto \phi_{2}(t)$ to $\dot{x}=f(x)$ satisfies

$$
\left|\phi_{1}(t)-\phi_{2}(t)\right| \leq \beta\left(\left|\phi_{1}(0)-\phi_{2}(0)\right|, t\right)
$$

for each $t$ in the domain of definition of $\phi_{1}$ and $\phi_{2}$; see, e.g., $[1,2,3]$. The bound (1) states that, over their domain of definition, the distance between two solutions is upper bounded by a function of the difference between their initial conditions and also decreases as $t$ gets arbitrarily large (when the domain of definition of the solutions is unbounded to the right). More general forms of this notion in terms of Riemannian distances have been studied in the context of contraction theory; see, e.g., the study of contracting and nonexpansive flows in $[4,5,6]$, the local arguments in [7], and the regional results in [8] in the context of observer design. Due to often being misinterpreted as a property of convergent systems [9], the authors in [10] provide a rigorous comparison between incremental stability and the property of convergent systems, and conclude that, general, neither implies the other without extra conditions (uniform convergent systems on positively invariant sets are shown in [10, Theorem 8] to imply incremental stability as defined therein). Of particular interest are the necessary and sufficient conditions in terms of Lyapunov functions reported in [2] for a notion of incremental stability that requires the bound (1) to hold for every pair of solutions.

In recent years, incremental stability has received growing attention due to its use in a wide range of applications and its suitability to study problems in which pairs of trajectories have to converge to each other. Incremental stability-like properties have been used in the study of synchronization [11, 12, 13], observer design [8, 14], control design [3], symbolic models for nonlinear time-delay systems [15], model reduction [16], as well as the study

\footnotetext{
${ }^{2}$ Though not made explicitly in the literature, the notion in (1) is uniform with respect to initial conditions by virtue of the properties of $\mathcal{K} \mathcal{L}$-functions.
} 
of convergent systems [17]. Unfortunately, the incremental stability notions and associated results available in the literature do not apply to systems with variables that can change continuously and, at times, jump discretely (see details in Section 2). These systems, known as hybrid systems, are capable of modeling a wide range of complex dynamical systems, including robotic, automotive, and power systems as well as natural processes. The availability of an incremental stability notion for this class of systems would enable the study of similar properties for them as the current notion for continuous-time systems allows. To the best of our knowledge, the notion of incremental stability and its properties for hybrid systems have not been thoroughly studied before, only discussed briefly in [18] for a class of transition systems in the context of bisimulations.

\subsection{Contributions}

This paper considers the hybrid systems framework presented in [19], where the continuous dynamics (or flows) are modeled using differential equations, while the discrete dynamics (or jumps) are captured by difference equations. Specifically, a hybrid system is denoted by $\mathcal{H}$, has data $(C, f, D, g)$, and is defined by the hybrid equations given by

$$
\begin{gathered}
\dot{z}=f(z) \quad z \in C, \\
z^{+}=g(z) \quad z \in D,
\end{gathered}
$$

where $z \in \mathbb{R}^{n}$ is the state, $f$ defines the flow map capturing the continuous dynamics, and $C$ defines the flow set on which $f$ is effective. The map $g$ defines the jump map and models the discrete behavior, while $D$ defines the jump set, which is the set of points from where jumps are allowed. While setvalued flow and jump maps could certainly be considered in (2), which would lead to hybrid inclusions, there is no loss of generality in considering singlevalued maps since, as it will be shown in Section 3, uniqueness of solutions (of their $x$ component) is a necessary condition for incremental stability; see Lemma 3.2. It should be noted that a robust stability theory for hybrid systems modeled as hybrid inclusions was developed in [19].

Motivated by the broad application of incremental stability and the recent advancements in the theory of hybrid systems given as in (2), incremental stability notions are introduced, and many of their basic properties, characterizations, and equivalences are presented. As will be discussed in Section 2, the jumps imposed by the difference equations make it inherently difficult to 
define incremental stability for hybrid systems. We address this issue by prioritizing flow time in the definition of incremental stability. More precisely, for a class of hybrid systems, the contributions of this paper include the following:

1) Notions of incremental stability: Non-uniform and uniform incremental (global) asymptotic stability notions with respect to full or partial state in terms of stability and attractivity are given.

2) Equivalent characterization of incremental stability: An equivalent characterization of incremental uniform (global) asymptotic stability in terms of a $\mathcal{K} \mathcal{L}$ bound on the distance between every two maximal solutions ${ }^{3}$ is provided.

3) Equivalent auxiliary system for the study of incremental stability through asymptotic stability of the diagonal-like set: An auxiliary hybrid system with twice the dimension of the original system is defined and the asymptotic stability properties of the diagonal-like set for the auxiliary system are related to incremental stability of the original system.

4) Relationships between incremental asymptotic stability and asymptotic stability properties: Links between properties involved in the definition of incremental asymptotic stability and of asymptotic stability of a system are revealed. In particular, it is shown that uniform global attractivity of a singleton set implies incremental uniform attractivity, and that strong forward invariance of a compact set plus uniform incremental stability of a system leads to uniform global asymptotic stability of the said set for a hybrid system with regular enough data.

This article contains new incremental stability results for the hybrid framework in [19]. A preliminary version of these results is shown in [20]. We

\footnotetext{
${ }^{3}$ A solution $\phi$ to $\mathcal{H}$ is parametrized by $(t, j) \in \mathbb{R}_{>0} \times \mathbb{N}$, where $t$ denotes ordinary time and $j$ denotes jump time. The domain of a solution dom $\phi \subset \mathbb{R}_{\geq 0} \times \mathbb{N}$ is a hybrid time domain if for every $(T, J) \in \operatorname{dom} \phi$, the set $\operatorname{dom} \phi \cap([0, T] \times\{0,1, \ldots, J\})$ can be written as the union of sets $\cup_{j=0}^{J}\left(I_{j} \times\{j\}\right)$, where $I_{j}:=\left[t_{j}, t_{j+1}\right]$ for some time sequence $0=t_{0} \leq t_{1} \leq t_{2} \leq \cdots \leq t_{J+1}$. The $t_{j}$ 's with $j>0$ define the time instants when the state of the hybrid system jumps and $j$ counts the number of jumps. A solution to $\mathcal{H}$ is called maximal if it cannot be extended, i.e., it is not a truncated version of another solution. It is called complete if its domain is unbounded.
} 
are not aware of any other previous results on incremental stability for hybrid systems (other than the discussions in [18] we mentioned in Section 1.1).

\subsection{Organization and Notation}

The remainder of this paper is organized as follows. In Section 2, the incremental stability notions are introduced for a class of hybrid systems. The auxiliary hybrid system is also introduced in Section 2. In Section 3, the main results are presented, namely, items 2)-4) listed in Section 1.2.

The notation used throughout the paper is as follows. Given a set $S \subset \mathbb{R}^{n}$, the closure of $S$ is denoted by $\bar{S}$, and the interior of $S$ is denoted by int $S$. The set of real and natural numbers are $\mathbb{R}_{\geq 0}:=[0, \infty)$ and $\mathbb{N}:=\{0,1, \ldots\}$, respectively. Given vectors $\nu \in \mathbb{R}^{n}, w \in \mathbb{R}^{m},|\nu|$ defines the Euclidean vector norm $|\nu|=\sqrt{\nu^{\top} \nu}$, and $\left[\nu^{\top} w^{\top}\right]^{\top}$ is equivalent to $(\nu, w)$. Given a function $f: \mathbb{R}^{m} \rightarrow \mathbb{R}^{n}$, its domain of definition is denoted by $\operatorname{dom} f$, i.e., dom $f:=\left\{x \in \mathbb{R}^{m}: f(x)\right.$ is defined $\}$. The range of $f$ is denoted by rge $f$, i.e., rge $f:=\{f(x): x \in \operatorname{dom} f\}$. The right limit of the function $f$ is defined as $f^{+}(x):=\lim _{\nu \rightarrow 0^{+}} f(x+\nu)$ if it exists. Given a point $y \in \mathbb{R}^{n}$ and a closed set $\mathcal{A} \subset \mathbb{R}^{n},|y|_{\mathcal{A}}:=\inf _{x \in \mathcal{A}}|x-y|$. A function $\alpha: \mathbb{R}_{\geq 0} \rightarrow \mathbb{R}_{\geq 0}$ is a class- $\mathcal{K}_{\infty}$ function, also written $\alpha \in \mathcal{K}_{\infty}$, if $\alpha$ is zero at zero, continuous, strictly increasing, and unbounded. A function $\beta: \mathbb{R}_{\geq 0} \times \mathbb{R}_{\geq 0} \rightarrow \mathbb{R}_{\geq 0}$ is a class- $\mathcal{K} \mathcal{L}$ function, also written $\beta \in \mathcal{K} \mathcal{L}$, if it is nondecreasing in its first argument, nonincreasing in its second argument, $\lim _{r \rightarrow 0^{+}} \beta(r, s)=0$ for each $s \in \mathbb{R}_{\geq 0}$, and $\lim _{s \rightarrow \infty} \beta(r, s)=0$ for each $r \in \mathbb{R}_{\geq 0}$.

\section{Incremental stability notions}

In this paper, for hybrid systems $\mathcal{H}$ as in (2), we are interested in characterizing the incremental stability property, namely, the notion that the distance between every pair of maximal solutions to the system has stable behavior and approaches zero asymptotically. To highlight the intricacies of this property in the hybrid setting, consider the so-called bouncing ball system. This is a canonical example of hybrid systems to which every maximal solution is Zeno ${ }^{4}$ and converges to the origin; see [19, Example 1.1 and 2.12] for more details. Consider two solutions to this system, given by $\phi_{1}$ and $\phi_{2}$, from initial conditions $\phi_{1}(0,0)=(1,0)$ (ball initialized close to the ground

\footnotetext{
${ }^{4} \mathrm{~A}$ solution is Zeno if it is complete and its domain is bounded in the $t$ direction. See [19] for more details.
} 


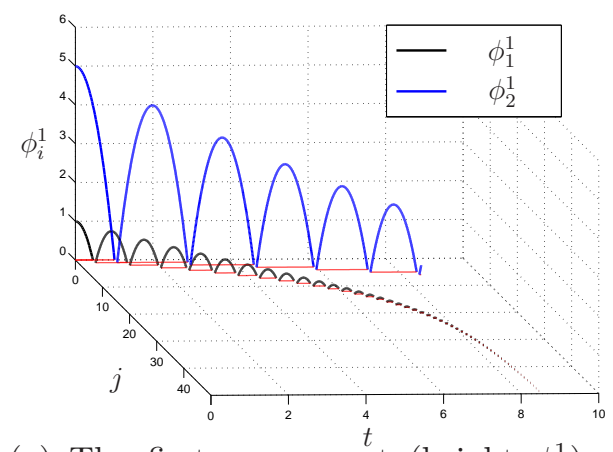

(a) The first component (height $\phi_{i}^{1}$ ) of solutions from $\phi_{1}(0,0)=(0,1)$ and $\phi_{2}(0,0)=(5,0)$.
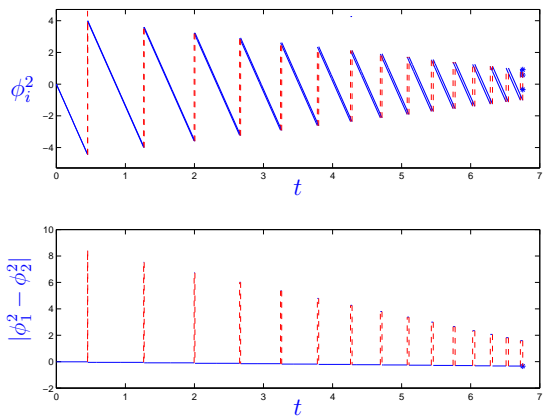

(b) Top: The second component (velocity) of solutions from $\phi_{1}(0,0)=(1,0)$ and $\phi_{2}(0,0)=(1.01,0.01)$. Bottom: The difference on the second component $\left|\phi_{1}^{2}-\phi_{2}^{2}\right|$ which exhibits peaking phenomenon.

Figure 1: Two solutions $\phi_{1}$ and $\phi_{2}$ to the bouncing ball system. Note that while both solutions converge to zero, the pointwise Euclidean distance between them does not decrease monotonically.

with zero initial velocity) and $\phi_{2}(0,0)=(5,0)$ (ball initialized at a larger height). Figure 1(a) shows the position (first) component ( $\phi_{i}^{1}$ for $i \in\{1,2\}$ ) of these two solutions. The Zeno behavior of the solutions makes it extremely difficult to analyze incremental stability. In fact, since incremental stability requires comparing the distance between two solutions, if one solution $\left(\phi_{1}\right)$ reaches the Zeno time sooner than the second solution $\left(\phi_{2}\right)$, then the Euclidean distance between these two solutions cannot be evaluated after one of the solutions has approached the Zeno time. This situation is shown in Figure 1(a), where $\phi_{1}$ approaches Zeno at about $t=8.5 \mathrm{sec}$ while $\phi_{2}$ is still describing the motion of the ball bouncing. Even if the solutions start from nearby initial conditions, the point-wise distance between them is not monotonically decreasing before the Zeno time. Figure 1(b) shows two solutions to the bouncing ball system with nearby initial conditions, but the offset on impact times creates a peaking behavior in the velocity error. In a more extreme situation, if solutions $\phi_{1}$ and $\phi_{2}$ are initialized at $\phi_{1}(0,0)=(0,0)$ and $\phi_{2}(0,0)=(1,0)$, respectively, then $\phi_{1}$ is a solution that only jumps and never evolves in the $t$ direction, while $\phi_{2}$ evolves in both directions ( $t$ and $j$ ) until it reaches a Zeno solution. This extreme difference in the domains of the solutions makes it difficult (if not impossible) to compare $\phi_{1}$ and $\phi_{2}$.

To avoid such issues, we consider the class of hybrid systems satisfying 
the following assumption.

Assumption 2.1. The hybrid system $\mathcal{H}=(C, f, D, g)$ is such that

1) each maximal solution to $\mathcal{H}$ has a hybrid time domain that is unbounded in the $t$ direction;

2) each solution to $\mathcal{H}$ does not have two consecutive jumps without flow in between.

Note that the solutions to a hybrid system $\mathcal{H}$ are parameterized by both $t$ and $j$, but we will consider incremental stability notions with bounds on the distance between solutions (like in (1)) that only depend on $t$. While such bounds might be conservative as they do not involve $j$, due to the general issue illustrated in the bouncing ball system, at this time it is not obvious how to define a notion of incremental stability for generic hybrid systems. The notions of incremental stability we consider in this paper are given as follows.

Definition 2.2. Consider a hybrid system $\mathcal{H}$ with state $z \in \mathbb{R}^{n}$ satisfying Assumption 2.1. Let $z=(x, \nu)$, where $x \in \mathbb{R}^{r}, \nu \in \mathbb{R}^{n-r}$, and $r, n$ are integers such that $1 \leq r \leq n$. The hybrid system $\mathcal{H}$ is said to be

- incrementally stable $(\delta \mathrm{S})$ with respect to $x$ if for every $\varepsilon>0$ there exists $\delta>0$ such that, for any two maximal solutions ${ }^{5} \phi_{1}=\left(\phi_{1}^{x}, \phi_{1}^{\nu}\right)$ and $\phi_{2}=\left(\phi_{2}^{x}, \phi_{2}^{\nu}\right)$ to $\mathcal{H}$,

$$
\left|\phi_{1}(0,0)-\phi_{2}(0,0)\right| \leq \delta
$$

implies $^{6}$

$$
\left|\phi_{1}^{x}\left(t, j_{1}(t)\right)-\phi_{2}^{x}\left(t, j_{2}(t)\right)\right| \leq \varepsilon
$$

for all $\left(t, j_{i}(t)\right) \in \operatorname{dom} \phi_{i}, i \in\{1,2\}$;

- incrementally globally attractive $(\delta \mathrm{GA})$ with respect to $x$ if any two maximal solutions $\phi_{1}=\left(\phi_{1}^{x}, \phi_{1}^{\nu}\right)$ and $\phi_{2}=\left(\phi_{2}^{x}, \phi_{2}^{\nu}\right)$ to $\mathcal{H}$ satisfy

$$
\lim _{\substack{t \rightarrow \infty \\\left(t, j_{1}(t)\right) \in \operatorname{dom} \phi_{1} \\\left(t, j_{2}(t)\right) \in \operatorname{dom} \phi_{2}}}\left|\phi_{1}^{x}\left(t, j_{1}(t)\right)-\phi_{2}^{x}\left(t, j_{2}(t)\right)\right|=0 ;
$$

\footnotetext{
${ }^{5}$ Given a solution $\phi$ to $\mathcal{H}$, we partition it as $\phi=\left(\phi^{x}, \phi^{\nu}\right)$, where $\phi^{x} \in \mathbb{R}^{r}$ and $\phi^{\nu} \in \mathbb{R}^{n-r}$.

${ }^{6}$ For each $t \in \mathbb{R}_{\geq 0}, t \mapsto j_{i}(t):=\min _{(t, j) \in \operatorname{dom} \phi_{i}} j$ for each $i \in\{1,2\}$.
} 
- incrementally globally asymptotically stable $(\delta \mathrm{GAS})$ with respect to $x$ if it is both $\delta S$ and $\delta G A$ with respect to $x$.

Remark 2.3. If $r=n$, then the notion in Definition 2.2 can be considered as a full-state incremental stability notion, while if $r<n$, it can be considered to be a partial state incremental stability notion. Note that $\delta S$ with respect to $x$ requires $\phi_{1}$ and $\phi_{2}$ to start close, while only the components $\phi_{1}^{x}$ and $\phi_{2}^{x}$ remain close over their domain of definition. Similarly, $\delta G A$ with respect to $x$ only requires the distance between $\phi_{1}^{x}$ and $\phi_{2}^{x}$ to approach zero, while the other components are left unconstrained.

Remark 2.4. Note that from the definition of $\delta S$, for each $i \in\{1,2\}$, the function $t \mapsto j_{i}(t)$ picks the minimum value $j$ for each $t$ such that $(t, j) \in$ dom $\phi_{i}$. Then, the resulting function $t \mapsto \phi_{i}\left(t, j_{i}(t)\right)$ is single valued and left continuous.

The following examples illustrate the incremental stability notions in Definition 2.2 .

Example 2.5. (system with a logic variable) Consider a hybrid system with state $z=(x, q) \in[-1,1] \times\{-1,1\}$ and with $n=2, r=1$, and data:

$$
\begin{array}{ll}
f(z):=(q, 0) & \forall z \in C:=[-1,1] \times\{-1,1\}, \\
g(z):=(x,-q) & \forall z \in D:=(\{-1\} \times\{-1\}) \cup(\{1\} \times\{1\}) .
\end{array}
$$

From the data of $\mathcal{H}$, Assumption 2.1 is satisfied. In fact, every maximal solution to $\mathcal{H}$ has a hybrid time domain that is unbounded in the $t$ and $j$ directions. Furthermore, solutions do not have two consecutive jumps without flow time in between since, in particular, $D \cap g(D)=\emptyset$ and the data of $\mathcal{H}$ is regular enough; see Lemma 3.1 for more details. Moreover, it can be verified that the system is $\delta S$ with respect to $x$; see Appendix $B$ for details.

Example 2.6. (system with a timer) Consider a hybrid system with state $z=(x, q, \tau, k) \in \mathcal{X}:=\mathbb{R} \times[-M, M] \times \mathbb{R}_{\geq 0} \times \mathbb{N} \backslash\{0\}$ for $M>0$ with $n=4$, $r=1$, and data:

$$
\begin{array}{ll}
f(z)=(-x+q, 0,1,0) & \forall z \in C:=\{z \in \mathcal{X}: \tau \leq k\}, \\
g(z)=(-x,-\gamma q, 0, k+1) & \forall z \in D:=\{z \in \mathcal{X}: \tau=k\},
\end{array}
$$


where the parameter $\gamma \in(0,1)$. From the data of $\mathcal{H}$, Assumption 2.1 is satisfied. In fact, every maximal solution to $\mathcal{H}$ has a hybrid time domain that is unbounded in the $t$ direction. Furthermore, solutions do not have two consecutive jumps without flow time in between since, in particular, $D \cap$ $g(D)=\emptyset$ and the data of $\mathcal{H}$ is regular enough; see Lemma 3.1. For a maximal solution $\phi$ to the system from $\phi(0,0)=\left(\phi^{x}(0,0), \phi^{q}(0,0), \phi^{\tau}(0,0), \phi^{k}(0,0)\right)$, the $\phi^{x}$ component of the solution before the first jump is given by $\phi^{x}(t, 0)=$ $\exp (-t) \phi^{x}(0,0)+(1-\exp (-t)) \phi^{q}(0,0)$ for all $t \in\left[0, \phi^{k}(0,0)-\phi^{\tau}(0,0)\right]$. Moreover, generalizing this expression to each $\left(\bar{t}_{j}, j\right)$ such that $\left(\bar{t}_{j}, j+1\right) \in$ $\operatorname{dom} \phi$, we obtain $\phi^{x}(t, j+1)=\exp \left(-t+\bar{t}_{j}\right) \phi^{x}\left(\bar{t}_{j}, j+1\right)+(1-\exp (-t+$ $\left.\left.\bar{t}_{j}\right)\right) \phi^{q}\left(\bar{t}_{j}, j+1\right)$ for each $t \in\left[\bar{t}_{j}, \phi^{k}\left(\bar{t}_{j}, j+1\right)-\phi^{\tau}\left(\bar{t}_{j}, j+1\right)\right]$, where $\phi\left(\bar{t}_{j}, j+1\right)=$ $g\left(\phi\left(\bar{t}_{j}, j\right)\right)$. Due to the form of the jump map for $q$, it follows that

$$
\lim _{\substack{j \rightarrow \infty \\\left(\bar{t}_{j}, j+1\right) \in \operatorname{dom} \phi}} \phi^{q}\left(\bar{t}_{j}, j+1\right)=0
$$

Since $j \rightarrow \infty$ if and only if $\bar{t}_{j} \rightarrow \infty$, we have

$$
\lim _{\substack{t \rightarrow \infty \\(t, j) \in \operatorname{dom} \phi}} \phi^{x}(t, j)=0
$$

Furthermore, if we have two solutions $\phi_{1}, \phi_{2}$ to $\mathcal{H}$ where $\phi_{i}=\left(\phi_{i}^{x}, \phi_{i}^{q}, \phi_{i}^{\tau}, \phi_{i}^{k}\right)$, $i \in\{1,2\}$ then

$$
\lim _{\substack{t \rightarrow \infty \\\left(t, j_{1}\right) \in \operatorname{dom} \phi_{1} \\\left(t, j_{2}\right) \in \operatorname{dom} \phi_{2}}}\left|\phi_{1}^{x}\left(t, j_{1}\right)-\phi_{2}^{x}\left(t, j_{2}\right)\right| \leq \lim _{\substack{t \rightarrow \infty \\\left(t, j_{1}\right) \in \operatorname{dom} \phi_{1} \\\left(t, j_{2}\right) \in \operatorname{dom} \phi_{2}}}\left(\left|\phi_{1}^{x}\left(t, j_{1}\right)\right|+\left|\phi_{2}^{x}\left(t, j_{2}\right)\right|\right)=0 .
$$

Therefore, the system is $\delta G A$ with respect to $x$.

As we will show, this system is not $\delta S$ with respect to $x$. To draw a contradiction, assume that it has such property. For a given $\varepsilon>0$, no matter how small $\delta>0$ is chosen, we always can pick two solutions $\phi_{i}=\left(\phi_{i}^{x}, \phi_{i}^{q}, \phi_{i}^{\tau}, \phi_{i}^{k}\right)$ from $\phi_{i}(0,0)=\left(\phi_{i}^{x}(0,0), \phi_{i}^{q}(0,0), \phi_{i}^{\tau}(0,0), \phi_{i}^{k}(0,0)\right)$ for $i \in\{1,2\}$, respectively, where $\phi_{1}^{q}(0,0)=\phi_{2}^{q}(0,0)=0, \phi_{1}^{k}(0,0)=\phi_{2}^{k}(0,0)>\phi_{1}^{\tau}(0,0)>$ $\phi_{2}^{\tau}(0,0)>0$ and $\phi_{1}^{x}(0,0)=\phi_{2}^{x}(0,0)>\frac{1}{2} \exp \left(\bar{t}_{1}\right) \varepsilon$ with $\bar{t}_{1}=\phi_{1}^{k}(0,0)-\phi_{1}^{\tau}(0,0)$. Moreover, $\phi_{1}(0,0)$ and $\phi_{2}(0,0)$ satisfy $\left|\phi_{1}(0,0)-\phi_{2}(0,0)\right| \leq \delta$. Then, we have that for all $0 \leq t \leq \bar{t}_{1}=\phi_{1}^{k}(0,0)-\phi_{1}^{\tau}(0,0)$ and for each $i \in\{1,2\}$, $\phi_{i}^{x}(t, 0)=\exp (-t) \phi_{i}^{x}(0,0)$. Then, after the first jump ( $\phi_{1}$ jumps first in this 
case), we have

$$
\begin{aligned}
\left|\phi_{1}^{x}\left(\bar{t}_{1}, 1\right)-\phi_{2}^{x}\left(\bar{t}_{1}, 0\right)\right| & =\exp \left(-\bar{t}_{1}\right)\left|-\phi_{1}^{x}(0,0)-\phi_{2}^{x}(0,0)\right| \\
& >\exp \left(-\bar{t}_{1}\right)\left|\frac{1}{2} \exp \left(\bar{t}_{1}\right) \varepsilon+\frac{1}{2} \exp \left(\bar{t}_{1}\right) \varepsilon\right|>\varepsilon,
\end{aligned}
$$

which is a contradiction.

Example 2.7. Consider the hybrid system in Example 2.6, but define the jump map as $g(z)=(x,-\gamma q, 0, k+1)$ for each $z \in D$. In this way, we can now show that this new system is $\delta G A S$ with respect to $x$. The proofs can be found in Appendix $C$.

Remark 2.8. Due to the definition of incremental stability, different choices of the component decompositions $(x, \nu)$ could lead to different conclusions about $\delta S$ and $\delta G A$. For instance, in Example 2.6, if the state component of interest is $q$, it can be shown that the system is also $\delta G A$ with respect to $q$, but not $\delta S$ with respect to $q$.

Building from Definition 2.2, a global and uniform notion of incremental asymptotic stability is introduced next.

Definition 2.9. Consider a hybrid system $\mathcal{H}$ with state $z \in \mathbb{R}^{n}$ satisfying Assumption 2.1. Let $z=(x, \nu)$, where $x \in \mathbb{R}^{r}, \nu \in \mathbb{R}^{n-r}$ and $n, r$ are integers such that $1 \leq r \leq n$. The hybrid system $\mathcal{H}$ is said to be

- incrementally uniformly globally stable ( $\delta \mathrm{UGS})$ with respect to $x$ if there exists a function $\alpha \in \mathcal{K}_{\infty}$ such that any two maximal solutions $\phi_{1}=\left(\phi_{1}^{x}, \phi_{1}^{\nu}\right)$ and $\phi_{2}=\left(\phi_{2}^{x}, \phi_{2}^{\nu}\right)$ to $\mathcal{H}$ satisfy

$$
\left|\phi_{1}^{x}\left(t, j_{1}(t)\right)-\phi_{2}^{x}\left(t, j_{2}(t)\right)\right| \leq \alpha\left(\left|\phi_{1}(0,0)-\phi_{2}(0,0)\right|\right)
$$

for all $\left(t, j_{1}(t)\right) \in \operatorname{dom} \phi_{1}$ and $\left(t, j_{2}(t)\right) \in \operatorname{dom} \phi_{2}$;

- incrementally uniformly globally attractive ( $\delta \mathrm{UGA})$ with respect to $x$ if for each $\varepsilon>0$ and $\bar{r}>0$ there exists $T>0$ such that for any two maximal solutions $\phi_{1}=\left(\phi_{1}^{x}, \phi_{1}^{\nu}\right)$ and $\phi_{2}=\left(\phi_{2}^{x}, \phi_{2}^{\nu}\right)$ to $\mathcal{H}$ with

$$
\left|\phi_{1}(0,0)-\phi_{2}(0,0)\right| \leq \bar{r}
$$

$\left(t, j_{i}(t)\right) \in \operatorname{dom} \phi_{i}$ for each $i \in\{1,2\}, t \geq T$ implies that

$$
\left|\phi_{1}^{x}\left(t, j_{1}(t)\right)-\phi_{2}^{x}\left(t, j_{2}(t)\right)\right| \leq \varepsilon ;
$$


- incrementally uniformly globally asymptotically stable ( $\delta$ UGAS) with respect to $x$ if it is both $\delta U G S$ and $\delta U G A$ with respect to $x$.

Remark 2.10. When a hybrid system in (2) has a jump set $D=\emptyset$ and a flow set $C$ given by the entire state space, the resulting hybrid system reduces to a continuous-time system. Moreover, if $r=n$, the notion of $\delta U G A S$ in Definition 2.9 reduces to the notion for continuous-time systems as in [2, Definition 2.1].

Example 2.11. Consider a hybrid system $\mathcal{H}$ with data

$$
\begin{array}{ll}
f(z):=-z & \forall z \in C:=\bigcup_{i \in\{2 k: k \in \mathbb{N}\}}[i, i+1] \\
g(z):=z-1 & \forall z \in D:=\{2 k: k \in \mathbb{N} \backslash\{0\}\} .
\end{array}
$$

Note that every maximal solution to $\mathcal{H}$ is complete, bounded and does not have two consecutive jumps without flow time in between since, in particular, $D \cap g(D)=\emptyset$ and the data of $\mathcal{H}$ is regular enough; Given $\varepsilon, \bar{r}>0$, consider two maximal solutions $\phi_{1}$ and $\phi_{2}$ from $\phi_{1}(0,0)=\xi$ and $\phi_{2}(0,0)=\eta$ such that $|\xi-\eta|<\bar{r}$, it follows that these solutions are bounded by

$$
\left|\phi_{i}\left(t, j_{i}\right)\right| \leq e^{-t}\left|\phi_{i}(0,0)\right|
$$

for all $\left(t, j_{i}(t)\right) \in$ dom $\phi_{i}$ and $i \in\{1,2\}$. Then, there exists $T>0$ such that $\left|\phi_{i}\left(t, j_{i}(t)\right)\right| \leq \frac{1}{2} \varepsilon$ for each $i \in\{1,2\}$ and each $t \geq T$ with $\left(t, j_{i}(t)\right) \in$ dom $\phi_{i}$. It follows that $\left|\phi_{1}\left(t, j_{1}(t)\right)-\phi_{2}\left(t, j_{2}(t)\right)\right| \leq \varepsilon$ for each $t \geq T$ with $\left(t, j_{i}(t)\right) \in$ dom $\phi_{i}$ with $i \in\{1,2\}$, which implies that this system is $\delta U G A$.

Following the approach in [2] for continuous-time systems, we can recast incremental stability as the problem of uniformly globally asymptotically stabilizing an appropriately defined hybrid system to the diagonal-like set. Towards that end, consider the set

$$
\tilde{\mathcal{A}}:=\left\{\tilde{z} \in \mathbb{R}^{n} \times \mathbb{R}^{n}: \tilde{z}=\left(\tilde{x}_{1}, \tilde{\nu}_{1}, \tilde{x}_{2}, \tilde{\nu}_{2}\right), \quad \tilde{x}_{1}=\tilde{x}_{2}, \quad \tilde{x}_{1}, \tilde{x}_{2} \in \mathbb{R}^{r}, \tilde{\nu}_{1}, \tilde{\nu}_{2} \in \mathbb{R}^{n-r}\right\}
$$


for the auxiliary hybrid system $\widetilde{\mathcal{H}}$ with state $\tilde{z}=\left(\tilde{z}_{1}, \tilde{z}_{2}\right) \in \mathbb{R}^{n} \times \mathbb{R}^{n}$ and given by the following data:

$$
\begin{gathered}
\dot{\tilde{z}}=\widetilde{f}(\tilde{z}) \quad \tilde{z} \in \widetilde{C}, \\
\tilde{z}^{+}=\widetilde{g}(\tilde{z}) \quad \tilde{z} \in \widetilde{D},
\end{gathered}
$$

where $\widetilde{C}:=C \times C, \widetilde{D}:=(D \times(C \cup D)) \cup((C \cup D) \times D), \tilde{f}(\tilde{z})=\left(f\left(\tilde{z}_{1}\right), f\left(\tilde{z}_{2}\right)\right)$, and $\widetilde{g}(\tilde{z})=\left(\widetilde{g}_{1}\left(\tilde{z}_{1}\right), \widetilde{g}_{2}\left(\tilde{z}_{2}\right)\right)$ with

$$
\begin{aligned}
& \widetilde{g}_{1}\left(\tilde{z}_{1}\right)= \begin{cases}g\left(\tilde{z}_{1}\right) & \tilde{z}_{1} \in D \\
\tilde{z}_{1} & \text { otherwise }\end{cases} \\
& \widetilde{g}_{2}\left(\tilde{z}_{2}\right)= \begin{cases}g\left(\tilde{z}_{2}\right) & \tilde{z}_{2} \in D \\
\tilde{z}_{2} & \text { otherwise }\end{cases}
\end{aligned}
$$

The auxiliary system $\widetilde{\mathcal{H}}$ consists of two copies of the original system $\mathcal{H}$.

Remark 2.12. Though the original hybrid system $\mathcal{H}$ may satisfy the socalled hybrid basic conditions ${ }^{7}$ in [19, Assumption 6.5], the auxiliary system $\widetilde{\mathcal{H}}$ may not. If the hybrid basic conditions were to be satisfied for $\widetilde{\mathcal{H}}$, the jump map would be set valued and have another entry given by $\widetilde{g}_{i}(\tilde{z})=\left\{g\left(\tilde{z}_{i}\right), \tilde{z}_{i}\right\}$ when $\tilde{z}_{i}$ is in the boundary of the jump set D. Including this entry in the jump map would not be advantageous since it could introduce extra solutions to the auxiliary system. In fact, it may introduce a Zeno solution: consider the case where $\tilde{z}_{1}$ and $\tilde{z}_{2}$ are in the boundary of the jump set; then, both components of the solution could jump to a point satisfying $\tilde{z} \in \widetilde{g}(\tilde{z}), \tilde{z} \in D \times D$ and remain there forever. To avoid this issue, we do not insist on $\widetilde{\mathcal{H}}$ satisfying the hybrid basic conditions.

It is worth noting that, in general, the domain of two solutions $\phi_{1}$ and $\phi_{2}$ of $\mathcal{H}$ with $\phi_{1}(0,0)=\xi$ and $\phi_{2}(0,0)=\eta$ are different from the domain of the associated solution $\tilde{\phi}$ of $\widetilde{\mathcal{H}}$ with $\tilde{\phi}(0,0)=(\xi, \eta)$. The following lemma characterizes the relationship (not necessarily incrementally stable) between solutions to $\mathcal{H}$ and solutions to $\widetilde{\mathcal{H}}$. Below, for a hybrid system $\mathcal{H}$, the set $\mathcal{S}_{\mathcal{H}}$ contains all maximal solutions to $\mathcal{H}$, and the set $\mathcal{S}_{\mathcal{H}}(\xi)$ contains all maximal solutions to $\mathcal{H}$ from $\xi$. The proof of this result is in Appendix A.

\footnotetext{
${ }^{7}$ A hybrid system $\mathcal{H}=(C, f, D, g)$ is said to satisfy the hybrid basic conditions if: (A1) $C$ and $D$ are closed; (A2) $f: \mathbb{R}^{n} \rightarrow \mathbb{R}^{n}$ and $g: \mathbb{R}^{n} \rightarrow \mathbb{R}^{n}$ are continuous; see [19, Assumption 6.5].
} 
Lemma 2.13. Suppose the hybrid system $\mathcal{H}$ satisfies Assumption 2.1. Then, the following hold:

1) Given a solution $\tilde{\phi} \in \mathcal{S}_{\widetilde{\mathcal{H}}}$ with $\tilde{\phi}(0,0)=(\xi, \eta), \xi, \eta \in \mathbb{R}^{n}$, there exist $\phi_{1}, \phi_{2} \in \mathcal{S}_{\mathcal{H}}, \phi_{1}(0,0)=\xi$ and $\phi_{2}(0,0)=\eta$ such that $\tilde{\phi}(t, j)=$ $\left(\phi_{1}\left(t, j_{1}\right), \phi_{2}\left(t, j_{2}\right)\right)$ for every $(t, j) \in \operatorname{dom} \tilde{\phi}$, where $\left(t, j_{1}\right) \in \operatorname{dom} \phi_{1}$ and $\left(t, j_{2}\right) \in$ dom $\phi_{2}$ for some $j_{1}, j_{2} \in \mathbb{N}$;

2) Given solutions $\phi_{\sim}, \phi_{2} \in \mathcal{S}_{\mathcal{H}}$ with $\phi_{1}(0,0)=\xi$ and $\phi_{2}(0,0)=\eta \underset{\sim}{\phi}, \xi, \eta \in$ $\mathbb{R}^{n}$, there exists $\tilde{\phi} \in \mathcal{S}_{\widetilde{\mathcal{H}}}$ with $\tilde{\phi}(0,0)=(\xi, \eta)$ such that $\tilde{\phi}(t, j)=$ $\left(\phi_{1}\left(t, j_{1}\right), \phi_{2}\left(t, j_{2}\right)\right)$ for every $\left(t, j_{1}\right) \in \operatorname{dom} \phi_{1}$ and $\left(t, j_{2}\right) \in \operatorname{dom} \phi_{2}$, where $(t, j) \in$ dom $\tilde{\phi}$ for some $j \in \mathbb{N}$.

In the next section, we provide our main results on incremental stability of $\mathcal{H}$, the stability of $\widetilde{\mathcal{A}}$ for $\widetilde{\mathcal{H}}$, and their relationships.

\section{Main results}

\subsection{Basic properties and their implications}

Due to the issues in defining incremental stability, the hybrid systems considered in this paper have to satisfy Assumption 2.1, which can be checked using Lemma 3.1 and [19, Proposition 6.10]. The following result from [21] provides a sufficient condition for condition 2) of Assumption 2.1.

Lemma 3.1. [21, Lemma 2.7] Suppose that $\mathcal{H}$ satisfies the hybrid basic conditions and $g(D) \cap D=\emptyset$. Then, for any precompact solution ${ }^{8} \phi \in \mathcal{S}_{\mathcal{H}}$ there exists $\gamma>0$ such that $\bar{t}_{j+1}-\bar{t}_{j} \geq \gamma$ for all $j \geq 1$, where $\left[\bar{t}_{j}, \bar{t}_{j+1}\right]=$ $\operatorname{dom} \phi \cap\left(\mathbb{R}_{\geq 0} \times\{j\}\right)$.

The following necessary condition for $\delta \mathrm{S}$ is immediate from its definition. While not pointed out in [2], the same property is necessary for incrementally stable continuous-time systems (where $r=n$ ).

Lemma 3.2. Suppose $\mathcal{H}$ satisfies Assumption 2.1 and is $\delta S$ with respect to $x$. Then, the projection of the $x$ component in the $t$ direction ${ }^{9}$ of every maximal solution $\phi=\left(\phi^{x}, \phi^{\nu}\right)$ to $\mathcal{H}$ is unique.

\footnotetext{
${ }^{8} \mathrm{~A}$ solution is precompact if it is complete and bounded.

${ }^{9}$ The projection of the $x$ component in the $t$ direction is defined as $t \mapsto \phi_{t}^{x}(t):=$ $\lim _{h \searrow 0,(t+h, j) \in \operatorname{dom} \phi} \phi(t+h, j)$.
} 
Proof We proceed by contradiction. Assume $\mathcal{H}$ is $\delta S$ with respect to $x$ and consider two maximal solutions $\phi_{1}=\left(\phi_{1}^{x}, \phi_{1}^{\nu}\right)$ and $\phi_{2}=\left(\phi_{2}^{x}, \phi_{2}^{\nu}\right)$ from $\phi_{1}(0,0)=\phi_{2}(0,0)=\xi \in \mathbb{R}^{n}$. Suppose that the projections of the $x$ component of these solutions in the $t$ direction are not unique, namely, there exists $t^{\star}>0$ such that $\phi_{t, 1}^{x}\left(t^{\star}\right) \neq \phi_{t, 2}^{x}\left(t^{\star}\right)$ and $\left(t^{\star}, j_{i}\right) \in \operatorname{dom} \phi_{i}, i \in\{1,2\}$. Pick $\varepsilon>0$ such that $\varepsilon<\left|\phi_{t, 1}^{x}\left(t^{\star}\right)-\phi_{t, 2}^{x}\left(t^{\star}\right)\right|$. Then, using this $\varepsilon$ in the $\delta \mathrm{S}$ property, no matter how small $\delta>0$ is chosen, we have that the solutions from $\phi_{1}(0,0)=$ $\phi_{2}(0,0)=\xi \in \mathbb{R}^{n}$, satisfy $\left|\phi_{t, 1}^{x}(t)-\phi_{t, 2}^{x}(t)\right|>\varepsilon$ when $t=t^{\star}$, which contradicts the definition of $\delta \mathrm{S}$.

Remark 3.3. Note that it is not necessary for the $\nu$ component of the solution to be unique. In fact, the bound proposed in (4) does not impose any restriction on the $\nu$ component. Lemma 3.2 implies that considering single-valued flow and jump maps in (2), rather than set-valued maps, is not restrictive. Furthermore, it precludes the possibility of solutions flowing from points in $C \cap D$.

Lemma 3.4. Suppose $\mathcal{H}$ satisfies Assumption 2.1 and the hybrid basic conditions. Then, $\widetilde{\mathcal{H}}$ does not have Zeno solutions.

Proof By Assumption 2.1 and [21, Lemma 2.7], for any solution to $\mathcal{H}$, the elapsed time between jumps is uniformly bounded below by a positive constant $(\gamma>0)$. Thus, for any solution $\tilde{\phi}$ to $\widetilde{\mathcal{H}}$, there exist at most two jumps on each time interval $[t, t+\gamma]$, for any $t \in\{t:(t, j) \in \operatorname{dom} \tilde{\phi}\}$. This can be proved using a similar contradiction argument as in the proof of Lemma 3.1. Then, it follows that there is no Zeno solution to $\widetilde{\mathcal{H}}$.

Motivated by Example 2.11, the next two results establish attractivity and stability properties of a set for $\mathcal{H}$ that are implied by $\delta \mathrm{GAS}$ of $\mathcal{H}$ with respect to $x$. For a hybrid system $\mathcal{H}$, a set $\mathcal{M} \subset \mathbb{R}^{n}$ is weakly forward invariant if for every $\xi \in \mathcal{M}$ there exists at least one complete solution $\phi \in \mathcal{S}_{\mathcal{H}}(\xi)$ with rge $\phi \subset \mathcal{M}$; see, e.g., [19, Definition 6.19]. The set $\mathcal{M}$ is strongly forward invariant if for every complete solution $\phi \in \mathcal{S}_{\mathcal{H}}(\mathcal{M})$, rge $\phi \subset \mathcal{M}$; see, e.g., [19, Definition 6.25]. Following [19, Definition 3.6], we consider the following definition of uniform global asymptotic stability (UGAS). 
Definition 3.5. Consider a hybrid system $\mathcal{H}$ on $\mathbb{R}^{n}$ with every $\phi \in \mathcal{S}_{\mathcal{H}}$ complete. Let $\mathcal{A} \subset \mathbb{R}^{n}$ be closed. The set $\mathcal{A}$ is said to be

- uniformly globally stable (UGS) for $\mathcal{H}$ if there exists a class $\mathcal{K}_{\infty}$ function $\alpha$ such that any solution $\phi$ to $\mathcal{H}$ satisfies $|\phi(t, j)|_{\mathcal{A}} \leq \alpha\left(|\phi(0,0)|_{\mathcal{A}}\right)$ for all $(t, j) \in \operatorname{dom} \phi$;

- uniformly globally attractive (UGA) for $\mathcal{H}$ if for each $\varepsilon>0$ and $r>0$ there exists $T>0$ such that, for any solution $\phi$ to $\mathcal{H}$ with $|\phi(0,0)|_{\mathcal{A}} \leq r$, $(t, j) \in \operatorname{dom} \phi$ and $t+j \geq T$ imply $|\phi(t, j)|_{\mathcal{A}} \leq \varepsilon ;$

- uniformly globally asymptotically stable (UGAS) for $\mathcal{H}$ if it is both uniformly globally stable and uniformly globally attractive.

Remark 3.6. Note that for a hybrid system $\mathcal{H}$ with state $z \in \mathbb{R}^{n}$ satisfying Assumption 2.1, every maximal solution to $\mathcal{H}$ is complete.

Theorem 3.7. Consider a hybrid system $\mathcal{H}$ on $\mathbb{R}^{n}$ with state $z=(x, \nu)$, $x \in \mathbb{R}^{r}, \nu \in \mathbb{R}^{n-r}$, where $n, r$ are integers such that $1 \leq r \leq n$, satisfying Assumption 2.1. Suppose that $\mathcal{H}$ is $\delta G A$ with respect to $x$. Then, a closed set $\mathcal{A}_{x} \times \mathbb{R}^{n-r} \subset \mathbb{R}^{r} \times \mathbb{R}^{n-r}$ that contains a nonempty weakly forward invariant set for $\mathcal{H}$ is globally attractive for $\mathcal{H}$.

Proof Assume $\mathcal{H}$ is $\delta$ GA with respect to $x$. Due to the fact that $\mathcal{A}_{x} \times \mathbb{R}^{n-r}$ contains a nonempty weakly forward invariant set, for each $\phi_{0}(0,0) \in \mathcal{A}_{x} \times$ $\mathbb{R}^{n-r}$, there exists a complete solution $\phi_{0}$ to $\mathcal{H}$ that satisfies $\phi_{0}(t, j(t)) \in$ $\mathcal{A}_{x} \times \mathbb{R}^{n-r}$ for all $(t, j(t)) \in \operatorname{dom} \phi_{0}$. Consider any maximal solution $\phi$ to $\mathcal{H}$. Since $\phi_{0}$ remains in $\mathcal{A}_{x} \times \mathbb{R}^{n-r}$, the distance between $\phi$ and $\mathcal{A}_{x} \times \mathbb{R}^{n-r}$ satisfies

$$
|\phi(t, j)|_{\mathcal{A}_{x} \times \mathbb{R}^{n-r}} \leq\left|\phi^{x}(t, j(t))-\phi_{0}^{x}\left(t, j_{0}(t)\right)\right|
$$

for all $(t, j(t)) \in \operatorname{dom} \phi$ and $\left(t, j_{0}(t)\right) \in \operatorname{dom} \phi_{0}$. Since $\mathcal{H}$ is incrementally globally attractive, we have that $\phi$ satisfies

$$
\lim _{\substack{t \rightarrow \infty \\(t, j(t)) \in \operatorname{dom} \phi \\\left(t, j_{0}(t)\right) \in \operatorname{dom} \phi_{0}}}\left|\phi^{x}(t, j(t))-\phi_{0}^{x}\left(t, j_{0}(t)\right)\right|=0
$$

which, from (10), implies that $\lim _{\substack{t \rightarrow \infty \\(t, j(t)) \in \operatorname{dom} \phi}}|\phi(t, j(t))|_{\mathcal{A}_{x} \times \mathbb{R}^{n-r}}=0$. Therefore, $\mathcal{A}_{x} \times \mathbb{R}^{n-r}$ is globally attractive for $\mathcal{H}$. 
Theorem 3.8. Consider $\mathcal{H}$ with $r=n$ satisfying Assumption 2.1 and is $\delta U G A S$. If $\mathcal{H}$ satisfies the hybrid basic conditions and the compact set $\mathcal{A} \subset \mathbb{R}^{n}$ is strongly forward invariant, then $\mathcal{A}$ is $U G A S$ for $\mathcal{H}$.

Proof Theorem 3.7 states that $\delta \mathrm{GA}$ with a closed set containing a weakly forward invariant set implies that the set is UGA for $\mathcal{H}$. Then, since $\mathcal{A} \subset \mathbb{R}^{n}$ is compact and strongly forward invariant, $\mathcal{A}$ is UGA for $\mathcal{H}$. Furthermore, since $\mathcal{H}$ satisfies the hybrid basic conditions, [19, Proposition 7.5] implies that $\mathcal{A}$ is stable. Hence, $\mathcal{A}$ is UGAS for $\mathcal{H}$.

Remark 3.9. For a hybrid system $\mathcal{H}$ on $\mathbb{R}^{n}$ with state $z=(x, \nu), x \in \mathbb{R}^{r}$, $\nu \in \mathbb{R}^{n-r}$, where $n, r$ are integers such that $1 \leq r \leq n$, the UGAS property guaranteed by Theorem 3.8 also holds when, instead of $\mathcal{A}$ being strongly forward invariant, $\mathcal{A}=\left\{x^{\star}\right\} \times \mathbb{R}^{n-r}$ with $x^{\star} \in \mathbb{R}^{r}$, which is a singleton set on the $x$ component.

\subsection{Equivalence properties}

The following result establishes a $\mathcal{K} \mathcal{L}$ characterization for $\delta$ UGAS. This notion is similar to that introduced in [2, Definition 2.1] for continuous-time systems.

Theorem 3.10. Consider a hybrid system $\mathcal{H}$ satisfying Assumption 2.1. Then, $\mathcal{H}$ is $\delta U G A S$ with respect to $x$ if and only if there exists a function $\beta \in \mathcal{K} \mathcal{L}$ such that for any two maximal solutions $\phi_{1}=\left(\phi_{1}^{x}, \phi_{1}^{\nu}\right)$ and $\phi_{2}=\left(\phi_{2}^{x}, \phi_{2}^{\nu}\right)$ to $\mathcal{H}$ from $\phi_{1}(0,0)=\xi$ and $\phi_{2}(0,0)=\eta$, respectively, where $x \in \mathbb{R}^{r}, \nu \in \mathbb{R}^{n-r}$ and $r, n$ are integers such that $1 \leq r \leq n$,

$$
\left|\phi_{1}^{x}\left(t, j_{1}(t)\right)-\phi_{2}^{x}\left(t, j_{2}(t)\right)\right| \leq \beta(|\xi-\eta|, t)
$$

for all $\left(t, j_{i}(t)\right) \in$ dom $\phi_{i}, i \in\{1,2\}$. Furthermore, under the $\delta U G A S$ assumption for $\mathcal{H}$, a continuous $\mathcal{K} \mathcal{L}$ function $\beta$ is guaranteed to exist.

Proof The sufficiency part follows immediately by the property of $\beta$. To show necessity, assume $\mathcal{H}$ is $\delta$ UGAS with respect to $x$. Define a function $\beta_{0}: \mathbb{R}_{\geq 0} \times \mathbb{R}_{\geq 0} \rightarrow[-\infty, \infty)$ by

$$
\begin{aligned}
\beta_{0}(r, s)=\sup \left\{\left|\phi_{1}^{x}\left(t, j_{1}(t)\right)-\phi_{2}^{x}\left(t, j_{2}(t)\right)\right|\right. & \\
\phi_{1} \in S_{\mathcal{H}}(\xi), \phi_{2} & \left.\in S_{\mathcal{H}}(\eta),|\xi-\eta| \leq r, t \geq s\right\} .
\end{aligned}
$$


Then, the bound

$$
\left|\phi_{1}^{x}\left(t, j_{1}(t)\right)-\phi_{2}^{x}\left(t, j_{2}(t)\right)\right| \leq \beta_{0}(|\xi-\eta|, t) \quad \forall\left(t, j_{i}(t)\right) \in \operatorname{dom} \phi_{i}
$$

holds for each solution pairs $\phi_{1}, \phi_{2}$ to $\mathcal{H}$. Moreover, for each $s, r \mapsto \beta_{0}(r, s)$ is nondecreasing and for each $r, s \mapsto \beta_{0}(r, s)$, is nonincreasing. Moreover, $\beta_{0}(r, s) \leq \alpha(r)$ for all $r, s \geq 0$, where $\alpha$ comes from the uniform global incremental stability property of $\mathcal{H}$. Define $\tilde{\beta}: \mathbb{R}_{\geq 0} \times \mathbb{R}_{\geq 0} \rightarrow \mathbb{R}_{\geq 0}$ by

$$
\tilde{\beta}(r, s)=\max \left\{0, \beta_{0}(r, s)\right\} .
$$

Then, the function $(r, s) \mapsto \tilde{\beta}(r, s)$ is nondecreasing and, due to the properties

of $\alpha$, continuous in $r$, nonincreasing in $s, \lim _{r \rightarrow 0^{+}} \tilde{\beta}(r, s)=0$ for each $s \in$ $\mathbb{R}_{\geq 0}$ since $\tilde{\beta}(r, s) \leq \alpha(r)$. Finally, uniform global asymptotic incremental attractivity of $\mathcal{H}$ implies that $\lim _{s \rightarrow \infty} \tilde{\beta}(r, s)=0$ for each $r \geq 0$. Hence, the inequality (11) is satisfied with $\tilde{\beta}$. Note that $\tilde{\beta}$ can be majorized by a continuous function $\beta \in \mathcal{K} \mathcal{L}$. In fact, following [19, Lemma 3.41], for each $\lambda>0$ there exist class- $\mathcal{K}_{\infty}$ functions $\tilde{\alpha}_{1}, \tilde{\alpha}_{2}$ such that, for all $r, s \in \mathbb{R}_{\geq 0}$, $\tilde{\beta}(r, s) \leq \tilde{\alpha}_{1}^{-1}\left(\tilde{\alpha}_{2}(r) e^{-\lambda s}\right)=: \beta(r, s)$. Since $\tilde{\alpha}_{1}, \tilde{\alpha}_{2} \in \mathcal{K}_{\infty}$, the resulting class$\mathcal{K} \mathcal{L}$ function $\beta$ is continuous.

\subsection{From $G A S$ to $\delta G A S$}

Theorem 3.8 asserts that strong forward invariance of a compact set for a hybrid system $\mathcal{H}$ that is $\delta$ UGAS implies that the compact set is UGAS for $\mathcal{H}$. The observation in Remark 3.9 relaxes the forward invariance assumption for the case of singleton set in the $x$ component. In this section, we explore the other direction of these implications, namely, from GAS of a set for $\mathcal{H}$, or of $\widetilde{\mathcal{A}}$ for $\widetilde{\mathcal{H}}$, to $\delta \mathrm{GAS}$ of $\mathcal{H}$.

We begin with the case of a singleton set (in the $x$ component) that is uniformly globally attractive.

Lemma 3.11. Consider a hybrid system $\mathcal{H}$ on $\mathbb{R}^{n}$ with state $z=(x, \nu)$, $x \in \mathbb{R}^{r}, \nu \in \mathbb{R}^{n-r}$, where $n, r$ are integers such that $1 \leq r \leq n$, satisfying Assumption 2.1. Suppose that a set $\mathcal{A}=\left\{x^{\star}\right\} \times \mathbb{R}^{n-r}$ is $U G A$ for $\mathcal{H}$, where $x^{\star} \in \mathbb{R}^{r}$. Then, the system $\mathcal{H}$ is $\delta U G A$ with respect to $x$.

Proof Consider any two maximal solutions $\phi_{1}=\left(\phi_{1}^{x}, \phi_{1}^{\nu}\right)$ and $\phi_{2}=\left(\phi_{2}^{x}, \phi_{2}^{\nu}\right)$ to $\mathcal{H}$ from $\phi_{1}(0,0)=\xi$ and $\phi_{2}(0,0)=\eta$, where $\xi, \eta \in \mathbb{R}^{n}$. Pick $\varepsilon>0$. Since 
$\mathcal{A}$ is uniformly globally attractive for $\mathcal{H}$, there exists $T$ such that, for all $\left(t, j_{i}\right) \in \operatorname{dom} \phi_{i}$, if $t+j_{i} \geq T$, then,

$$
\left|\phi_{1}^{x}\left(t, j_{1}\right)-x^{\star}\right| \leq \frac{1}{2} \varepsilon, \quad\left|\phi_{2}^{x}\left(t, j_{2}\right)-x^{\star}\right| \leq \frac{1}{2} \varepsilon .
$$

Choose $j_{0}=\min \left\{j_{i}: t+j_{i} \geq T,\left(t, j_{i}\right) \in \operatorname{dom} \phi_{i}, i \in\{1,2\}\right\}$. Then, we have that for all $t \geq T-j_{0}$, (15) holds. Therefore, we have,

$$
\left|\phi_{1}^{x}\left(t, j_{1}(t)\right)-\phi_{2}^{x}\left(t, j_{2}(t)\right)\right| \leq \varepsilon \quad \forall t \geq T-j_{0},
$$

where $\left(t, j_{i}(t)\right) \in \operatorname{dom} \phi_{i}$, namely, $\mathcal{H}$ is $\delta \mathrm{UGA}$ with respect to $x$.

The incremental stability property can also be studied in terms of the auxiliary hybrid system $\widetilde{\mathcal{H}}$ introduced in Section 2. The following result establishes a relationship between GAS of a diagonal-like set for $\widetilde{\mathcal{H}}$ and $\delta \mathrm{GAS}$ for $\mathcal{H}$.

Theorem 3.12. Consider a hybrid system $\mathcal{H}$ on $\mathbb{R}^{n}$ with state $z=(x, \nu)$, $x \in \mathbb{R}^{r}, \nu \in \mathbb{R}^{n-r}$, where $n, r$ are integers such that $1 \leq r \leq n$, satisfying Assumption 2.1. Then, for the set $\widetilde{\mathcal{A}}$ in (7), the following hold:

i) $\mathcal{H}$ is $\delta G A S$ with respect to $x$ if $\widetilde{\mathcal{A}}$ is $G A S$ for the auxiliary hybrid system $\widetilde{\mathcal{H}}=(\widetilde{C}, \widetilde{f}, \widetilde{D}, \widetilde{g})$;

ii) $\mathcal{H}$ with $r=n$ is $\delta U G A$ with respect to $x$ if and only if $\widetilde{\mathcal{A}}$ is $U G A$ for $\widetilde{\mathcal{H}}$

iii) $\mathcal{H}$ with $r=n$ is $\delta G A S$ with respect to $x$ (uniformly) if and only if $\widetilde{\mathcal{A}}$ is GAS (respectively, uniformly) for $\widetilde{\mathcal{H}}$.

Proof Let $\xi, \eta \in \mathbb{R}^{n}$ and $\tilde{\phi}=\left(\tilde{\phi}_{1}, \tilde{\phi}_{2}\right)$ be a maximal solution to $\widetilde{\mathcal{H}}$ with $\tilde{\phi}_{i}=\left(\tilde{\phi}_{i}^{x}, \tilde{\phi}_{i}^{\nu}\right) \in \mathbb{R}^{n}$ and $\tilde{\phi}(0,0)=(\xi, \eta)$. Furthermore, by Lemma 2.13, there exists $\phi_{i}=\left(\phi_{i}^{x}, \phi_{i}^{\nu}\right) \in S_{\mathcal{H}}$ for $i \in\{1,2\}$ with $\phi_{1}(0,0)=\xi$ and $\phi_{2}(0,0)=\eta$. Inspired by the proof of [2, Lemma 2.3], we define a hybrid arc $\sigma^{\star}$ (not necessarily a solution) such that for each $(t, j(t)) \in \operatorname{dom} \tilde{\phi}$, we have

$$
\sigma^{\star}(t, j(t))=\frac{1}{2}\left(\tilde{\phi}_{1}^{x}(t, j(t))+\tilde{\phi}_{2}^{x}(t, j(t)), \tilde{\phi}_{1}^{x}(t, j(t))+\tilde{\phi}_{2}^{x}(t, j(t))\right)
$$


Moreover, the solution $\tilde{\phi}$ and the hybrid arc $\sigma^{\star}$ satisfy

$$
\begin{aligned}
|\tilde{\phi}(t, j(t))|_{\widetilde{\mathcal{A}}} & =\inf _{\sigma \in\left\{\left(\tilde{x}_{1}, \tilde{x}_{2}\right) \in \mathbb{R}^{r} \times \mathbb{R}^{r}: \tilde{x}_{1}=\tilde{x}_{2}\right\}}\left|\left(\tilde{\phi}_{1}^{x}(t, j(t)), \tilde{\phi}_{2}^{x}(t, j(t))\right)-\sigma\right| \\
& =\left|\left(\tilde{\phi}_{1}^{x}(t, j(t)), \tilde{\phi}_{2}^{x}(t, j(t))\right)-\sigma^{\star}\right| \\
& =\frac{\sqrt{2}}{2}\left|\phi_{1}^{x}\left(t, j_{1}(t)\right)-\phi_{2}^{x}\left(t, j_{2}(t)\right)\right|
\end{aligned}
$$

for every $t \in \mathbb{R}_{\geq 0}$ and some $j(t), j_{1}(t), j_{2}(t)$ such that $(t, j(t)) \in \operatorname{dom} \tilde{\phi}$ and $\left(t, j_{i}(t)\right) \in \operatorname{dom} \phi_{i}, i \in\{1,2\}$.

First, we show item i). Assume that $\widetilde{\mathcal{A}}$ is stable to $\widetilde{\mathcal{H}}$. Then, given $\varepsilon>0$, pick $\delta>0$ such that $|\tilde{\phi}(0,0)|_{\widetilde{\mathcal{A}}} \leq \delta$ implies $|\tilde{\phi}(t, j(t))|_{\widetilde{\mathcal{A}}} \leq \frac{1}{\sqrt{2}} \varepsilon$ for all $(t, j(t)) \in \operatorname{dom} \tilde{\phi}$ and $\left|\phi_{1}(0,0)-\phi_{2}(0,0)\right| \leq \delta$. Therefore, from (16) it follows that

$$
\left|\phi_{1}^{x}\left(t, j_{1}(t)\right)-\phi_{2}^{x}\left(t, j_{2}(t)\right)\right|=\sqrt{2}|\tilde{\phi}(t, j(t))|_{\widetilde{\mathcal{A}}} \leq \varepsilon
$$

for all $t \in \mathbb{R}_{\geq 0}$ and some $j(t), j_{1}(t), j_{2}(t)$ such that $\left(t, j_{i}(t)\right) \in \operatorname{dom} \phi_{i}$, $i \in\{1,2\}$, and hence $\mathcal{H}$ is $\delta \mathrm{S}$ with respect to $x$. Next, we show that $\mathcal{H}$ is $\delta$ GA with respect to $x$. Since $\widetilde{\mathcal{A}}$ is globally attractive for $\widetilde{\mathcal{H}}$, then for any solution $\tilde{\phi}$ to $\widetilde{\mathcal{H}}$, it follows that

$$
\lim _{\substack{t \rightarrow \infty \\(t, j(t)) \in \operatorname{dom} \tilde{\phi}}}|\tilde{\phi}(t, j(t))|_{\widetilde{\mathcal{A}}}=0 .
$$

From (16) and Lemma 2.13, it follows that

$\lim _{\substack{t \rightarrow \infty \\\left(t, j_{i}(t)\right) \in \operatorname{dom} \phi_{i}, i \in\{1,2\}}}\left|\phi_{1}^{x}\left(t, j_{1}(t)\right)-\phi_{2}^{x}\left(t, j_{2}(t)\right)\right|=\lim _{\substack{t \rightarrow \infty \\(t, j(t)) \in \operatorname{dom} \tilde{\phi}}} \sqrt{2}|\tilde{\phi}(t, j(t))|_{\tilde{\mathcal{A}}}=0$,

implying that $\mathcal{H}$ is $\delta \mathrm{GA}$ with respect to $x$. Therefore, $\mathcal{H}$ is $\delta \mathrm{GAS}$ with respect to $x$.

Now we show item ii). First, we show sufficiency. Assume that $\widetilde{\mathcal{A}}$ is UGA for $\widetilde{\mathcal{H}}$. Given $\varepsilon>0$ and $\bar{r}>0$, let $|\tilde{\phi}(0,0)|_{\widetilde{\mathcal{A}}} \leq \frac{1}{\sqrt{2}} \bar{r}$ and $\left|\phi_{1}(0,0)-\phi_{2}(0,0)\right| \leq \bar{r}$ then there exists $T>0$ such that for $t \geq T$ implies $|\tilde{\phi}(t, j(t))|_{\widetilde{\mathcal{A}}} \leq \frac{1}{\sqrt{2}} \varepsilon$. Therefore, from (16) with $r=n$, it follows that $\left|\phi_{1}\left(t, j_{1}(t)\right)-\phi_{2}\left(t, j_{2}(t)\right)\right|=$ $\sqrt{2}|\tilde{\phi}(t, j(t))|_{\widetilde{\mathcal{A}}} \leq \varepsilon$ for all $t \geq T$ and some $j(t), j_{1}(t), j_{2}(t)$ such that $\left(t, j_{i}(t)\right) \in \operatorname{dom} \phi_{i}, i \in\{1,2\}$. Then, $\mathcal{H}$ is $\delta \mathrm{UGA}$ with respect to $x$. Using the fact that, $r$ with $r=n,(16)$ leads to $|\tilde{\phi}(t, j(t))|_{\widetilde{\mathcal{A}}}=\frac{\sqrt{2}}{2} \mid \phi_{1}\left(t, j_{1}(t)\right)-$ 
$\phi_{2}\left(t, j_{2}(t)\right) \mid$ (full state equivalence) for all $t \in \mathbb{R}_{\geq 0}$ and some $j(t), j_{1}(t), j_{2}(t)$ such that $\left(t, j_{i}(t)\right) \in \operatorname{dom} \phi_{i}, i \in\{1,2\}$, necessity follows similarly.

Lastly, we show item iii). Since $\widetilde{\mathcal{A}}$ is UGAS for $\widetilde{\mathcal{H}}$, then by $[19$, Theorem 3.40] there exists $\beta \in \mathcal{K} \mathcal{L}$ such that

$$
|\tilde{\phi}(t, j(t))|_{\widetilde{\mathcal{A}}} \leq \beta\left(|\tilde{\phi}(0,0)|_{\widetilde{\mathcal{A}}}, t\right) .
$$

for every $(t, j(t)) \in \operatorname{dom} \tilde{\phi}$. From (16) with $r=n$, we have that

$$
\begin{aligned}
\left|\phi_{1}\left(t, j_{1}(t)\right)-\phi_{2}\left(t, j_{2}(t)\right)\right|=\sqrt{2}|\tilde{\phi}(t, j(t))|_{\widetilde{\mathcal{A}}} & \leq \sqrt{2} \beta\left(|\tilde{\phi}(0,0)|_{\widetilde{\mathcal{A}}}, t\right) \\
& \leq \sqrt{2} \beta\left(\frac{\sqrt{2}}{2}\left|\phi_{1}(0,0)-\phi_{2}(0,0)\right|, t\right)
\end{aligned}
$$

for all $t \in \mathbb{R}_{\geq 0}$ and some $j(t), j_{1}(t), j_{2}(t)$ such that $\left(t, j_{i}(t)\right) \in \operatorname{dom} \phi_{i}$, $i \in\{1,2\}$ and $(t, j(t)) \in \operatorname{dom} \tilde{\phi}$. Therefore, by Theorem 3.10, $\mathcal{H}$ is $\delta$ UGAS with respect to $x$. To show the other direction, note that when $r=n$, equation (16) becomes $|\tilde{\phi}(t, j(t))|_{\widetilde{\mathcal{A}}}=\frac{\sqrt{2}}{2}\left|\phi_{1}\left(t, j_{1}(t)\right)-\phi_{2}\left(t, j_{2}(t)\right)\right|$ (full state equivalence). In particular, given that $\mathcal{H}$ is $\delta \mathrm{UGAS}$ with respect to $x$ and two maximal solutions $\phi_{1}, \phi_{2}$ to $\mathcal{H}$, it follows from Theorem 3.10 that there exists $\bar{\beta} \in \mathcal{K} \mathcal{L}$ such that

$$
\begin{aligned}
|\tilde{\phi}(t, j(t))|_{\widetilde{\mathcal{A}}}=\frac{\sqrt{2}}{2}\left|\phi_{1}\left(t, j_{1}(t)\right)-\phi_{2}\left(t, j_{2}(t)\right)\right| & \leq \frac{\sqrt{2}}{2} \bar{\beta}\left(\left|\phi_{1}(0,0)-\phi_{2}(0,0)\right|, t\right) \\
& \leq \frac{\sqrt{2}}{2} \bar{\beta}\left(\sqrt{2}|\tilde{\phi}(0,0)|_{\widetilde{\mathcal{A}}}, t\right)
\end{aligned}
$$

for all $t \in \mathbb{R}_{\geq 0}$ and some $j(t), j_{1}(t), j_{2}(t)$ such that $\left(t, j_{i}(t)\right) \in \operatorname{dom} \phi_{i}$, $i \in\{1,2\}$ and $(t, j(t)) \in \operatorname{dom} \tilde{\phi}$. Therefore, by [19, Theorem 3.40], $\widetilde{\mathcal{H}}$ is UGAS. Note that the sufficiency of the non-uniform case is proved in item i); the necessity follows from (16) with $r=n,(17)$ and (18).

The following result provides a Lyapunov condition that guarantees the stability property of $\widetilde{\mathcal{A}}$ for $\widetilde{\mathcal{H}}$.

Theorem 3.13. [19, Theorem 3.18] Consider a hybrid system $\mathcal{H}$ on $\mathbb{R}^{n}$ with state $z \in \mathbb{R}^{n}$ satisfying Assumption 2.1. Suppose $V$ is a Lyapunov function candidate $^{10}$ for $\widetilde{\mathcal{H}}=(\widetilde{C}, \widetilde{f}, \widetilde{D}, \widetilde{g})$ in (8), and there exist $\alpha_{1}, \alpha_{2} \in \mathcal{K}_{\infty}$ and a

\footnotetext{
${ }^{10}$ See [19, Theorem 3.16] for a definition.
} 
positive definite continuous function $\rho$ such that

$$
\begin{aligned}
\alpha_{1}\left(|\tilde{z}|_{\tilde{\mathcal{A}}}\right) \leq V(\tilde{z}) \leq \alpha_{2}\left(|\tilde{z}|_{\widetilde{\mathcal{A}}}\right) & \forall \tilde{z} \in \widetilde{C} \cup \widetilde{D} \cup \widetilde{g}(\widetilde{D}), \\
\langle\nabla V(\tilde{z}), f(\tilde{z})\rangle \leq-\rho\left(|\tilde{z}|_{\widetilde{\mathcal{A}}}\right) & \forall \tilde{z} \in \widetilde{C} \\
V(g(\tilde{z}))-V(\tilde{z}) \leq-\rho\left(|\tilde{z}|_{\widetilde{\mathcal{A}}}\right) & \forall \tilde{z} \in \widetilde{D}
\end{aligned}
$$

Then, $\widetilde{\mathcal{A}}$ defined in (7) is UGAS for $\widetilde{\mathcal{H}}$. Furthermore, $\mathcal{H}$ is $\delta G A S$.

Remark 3.14. Relaxed versions of Theorem 3.13 can be found in [19, Propositions 3.24, 3.27, and 3.29]. Note that the sufficient conditions in (19) reduce to those for continuous-time systems as in [2, Remark 2.4] when $C=\mathbb{R}^{n}$ and $D=\emptyset$. Furthermore, the case when $C=\emptyset$ and $D=\mathbb{R}^{n}$ provides a version for discrete-time systems, which we are not aware of existing in the literature. The conditions in (19) are also illustrated in [20, Section VI] on what they require for hybrid systems.

The next result considers the case when a subset of $\widetilde{\mathcal{A}}$ is UGAS for $\widetilde{\mathcal{H}}$. Its proof is inspired by the ideas in the proof of Theorem 3.12.

Corollary 3.15. Consider a hybrid system $\mathcal{H}$ on $\mathbb{R}^{n}$ with state $z=(x, \nu)$, $x \in \mathbb{R}^{r}, \nu \in \mathbb{R}^{n-r}$, and $n, r$ are integers such that $1 \leq r \leq n$, satisfying Assumption 2.1. Then, $\mathcal{H}$ is $\delta G A$ with respect to $x$ if a nonempty closed set $\mathcal{A}_{s} \subset \widetilde{\mathcal{A}}$ is $G$ A for the auxiliary hybrid system $\widetilde{\mathcal{H}}=(\widetilde{C}, \widetilde{f}, \widetilde{D}, \widetilde{g})$.

Proof Consider two maximal solutions $\phi_{i}=\left(\phi_{i}^{x}, \phi_{i}^{\nu}\right) \in S_{\mathcal{H}}$ for $i \in\{1,2\}$ with $\phi_{1}(0,0)=\xi$ and $\phi_{2}(0,0)=\eta$. For each $\left(t, j_{i}\right) \in \operatorname{dom} \phi_{i}$, by Lemma 2.13, there exists $\tilde{\phi} \in S_{\widetilde{\mathcal{H}}}$ with $\tilde{\phi}(0,0)=(\xi, \eta)$ such that $\tilde{\phi}(t, j(t))=\left(\phi_{1}\left(t, j_{1}(t)\right), \phi_{2}\left(t, j_{2}(t)\right)\right)$. Since $\mathcal{A}_{s} \subset \widetilde{\mathcal{A}}$, then $|\tilde{\phi}(t, j)|_{\widetilde{\mathcal{A}}} \leq|\tilde{\phi}(t, j(t))|_{\mathcal{A}_{s}}$. Furthermore, since $\widetilde{\mathcal{H}}$ has $\mathcal{A}_{s}$ global attractive, $\left|\phi_{1}^{x}\left(t, j_{1}(t)\right)-\phi_{2}^{x}\left(t, j_{2}(t)\right)\right|=\sqrt{2}|\tilde{\phi}(t, j(t))|_{\widetilde{\mathcal{A}}} \leq \sqrt{2}|\tilde{\phi}(t, j(t))|_{\mathcal{A}_{s}}$ for every $t \in \mathbb{R}_{\geq 0}$ and some $j(t), j_{1}(t), j_{2}(t)$ such that $\left(t, j_{i}(t)\right) \in \operatorname{dom} \phi_{i}, i \in$ $\{1,2\}$ and $(t, j(t)) \in \operatorname{dom} \tilde{\phi}$, following similar steps as in the proof of Theorem 3.12, we have $\lim _{\substack{t \rightarrow \infty \\\left(t, j_{i}(t)\right) \in \operatorname{dom} \phi_{i}, i \in\{1,2\}}}\left|\phi_{1}^{x}\left(t, j_{1}(t)\right)-\phi_{2}^{x}\left(t, j_{2}(t)\right)\right|=0$. Thus, $\mathcal{H}$ is $\delta \mathrm{GA}$ with respect to $x$. 


\section{Conclusion}

Several notions of incremental stability for hybrid systems were introduced and studied. These notions prioritize the time parameter $t$ due to the disparity of the domain of the solutions to such systems. An equivalent $\mathcal{K} \mathcal{L}$ characterization of incremental stability as well as relationships between a set being asymptotically stable and a system being incrementally stable were also established. Existing results from the literature of hybrid systems, including those using Lyapunov functions, can already be applied to the proposed auxiliary hybrid system to certify incremental stability of the original system. Current efforts are focused on constructive sufficient conditions for incremental stability. We acknowledge that there are difficulties in relaxing these conditions for hybrid systems, and such relaxations are part of our current research.

\section{Acknowledgement}

This research has been partially supported by the National Science Foundation under CAREER Grant no. ECCS-1450484 and by the Air Force Office of Scientific Research under YIP Grant no. FA9550-12-1-0366.

\section{References}

[1] C. Feng, P. Wang, The existence of almost periodic solutions of some delay differential equations, Computers \& Mathematics with Applications 47 (8-9) (2004) $1225-1231$.

[2] D. Angeli, A Lyapunov approach to incremental stability properties, IEEE Transactions on Automatic Control 47 (3) (2002) 410-421.

[3] M. Zamani, P. Tabuada, Backstepping design for incremental stability, IEEE Transactions on Automatic Control 56 (9) (2011) 2184-2189.

[4] D. C. Lewis, Metric properties of differential equations, American Journal of Mathematics 71 (1949) 294-312.

[5] G. Isac, S. Z. Németh, Scalar and asymptotic scalar derivatives : theory and applications, 4th Edition, Springer, 2008. 
[6] F. Forni, R. Sepulchre, A differential lyapunov framework for contraction analysis, IEEE Transactions on Automatic Control 59 (3) (2014) 614628.

[7] W. Lohmiller, J.-J. Slotine, On contraction analysis for nonlinear systems, Automatica 34 (6) (1998) 671-682.

[8] R. G. Sanfelice, L. Praly, Convergence of nonlinear observers on $\mathbb{R}^{n}$ with a Riemannian metric (Part I), IEEE Transactions on Automatic Control 57 (7) (2012) 1709-1722.

[9] B. P. Demidovich, Dissipativity of a nonlinear system of differential equations, Ser. Mat; Mekh. Part I.6 (1961); Part II.1, 3-8(1962) (in Russian). 19-27.

[10] B. S. Ruffer, N. van de Wouw, M. Mueller, Convergent systems vs. incremental stability, Systems \& Control Letters 62 (3) (2013) 277-285.

[11] C. Cai, G. Chen, Synchronization of complex dynamical networks by the incremental ISS approach, Physica A: Statistical Mechanics and its Applications 371 (2) (2006) $754-766$.

[12] Q. Pham, N. Tabareau, J. J. Slotine, A contraction theory approach to stochastic incremental stability, IEEE Transactions on Automatic Control 54 (4) (2009) 816-820.

[13] A. Hamadeh, G. B. Stan, J. Goncalves, Constructive synchronization of networked feedback systems, in: Proc. 49th IEEE Conference on Decision and Control, 2010, pp. 6710-6715.

[14] A. P. Dani, S. J. Chung, S. Hutchinson, Observer design for stochastic nonlinear systems using contraction analysis, in: Proc. 51st Annual Conference on Decision and Control, 2012, pp. 6028-6035.

[15] G. Pola, P. Pepe, M. D. D. Benedetto, P. Tabuada, Symbolic models for nonlinear time-delay systems using approximate bisimulations, Systems \& Control Letters 59 (6) (2010) 365-373.

[16] B. Besselink, N. van de Wouw, H. Nijmeijer, Model reduction for nonlinear systems with incremental gain or passivity properties, Automatica 49 (4) (2013) 861-872. 
[17] A. Pavlov, N. van de Wouw, H. Nijmeijer, Frequency response functions for nonlinear convergent systems, IEEE Transactions on Automatic Control 52 (6) (2007) 1159-1165.

[18] P. Prabhakar, J. Liu, R. M. Murray, Pre-orders for reasoning about stability properties with respect to input of hybrid systems, in: In Proc. of the International Conference on Embedded Software (EMSOFT), 2013, pp. $1-10$.

[19] R. Goebel, R. G. Sanfelice, A. R. Teel, Hybrid Dynamical Systems: Modeling, Stability, and Robustness, Princeton University Press, New Jersey, 2012.

[20] Y. Li, S. Phillips, R. G. Sanfelice, Results on incremental stability for a class of hybrid systems, in: Proc. of IEEE 53rd Annual Conference on Decision and Control, 2014, pp. 3089-3094.

[21] R. G. Sanfelice, R. Goebel, A. R. Teel, Invariance principles for hybrid systems with connections to detectability and asymptotic stability, IEEE Transactions on Automatic Control 52 (12) (2007) 2282-2297.

\section{Appendix A. Proof of Lemma 2.13}

Proof Pick a maximal solution $\tilde{\phi} \in \mathcal{S}_{\tilde{\mathcal{H}}}$ with $\tilde{\phi}(0,0)=(\xi, \eta)$, where $\xi, \eta \in \mathbb{R}^{n}$. For some sequence $0=t_{0} \leq t_{1} \leq t_{2} \leq \ldots$, let $\operatorname{dom} \tilde{\phi}:=\bigcup_{j=0}^{\infty}\left(\left[t_{j}, t_{j+1}\right], j\right)$ if there are infinitely many jumps, otherwise let $\operatorname{dom} \tilde{\phi}:=\bigcup_{j=0}^{J-1}\left(\left[t_{j}, t_{j+1}\right], j\right) \cup$ $\left(\left[t_{J}, \infty\right), J\right)$. Then, by [19, Definition 2.6],

1) for each $j \in \mathbb{N}$ with $I_{j}=\left[t_{j}, t_{j+1}\right]$, and possibly of the form $I_{J}=\left[t_{J}, \infty\right)$ for $J=\sup _{(t, j) \in \operatorname{dom} \tilde{\phi}} j$, having a nonempty interior, $\tilde{\phi}(t, j)$ satisfies $\tilde{\phi}(t, j) \in C \times C$ for each $t \in \operatorname{int} I_{j}$, and $\dot{\tilde{\phi}}_{1}(t, j)=f\left(\tilde{\phi}_{1}(t, j)\right)$ and $\dot{\tilde{\phi}}_{2}(t, j)=f\left(\tilde{\phi}_{2}(t, j)\right)$ for almost all $t \in I_{j}$;

2) for all $(t, j) \in \operatorname{dom} \tilde{\phi}$ such that $(t, j+1) \in \operatorname{dom} \tilde{\phi}$, we have

a) if $\tilde{\phi}(t, j) \in D \times(C \backslash D)$, then $\tilde{\phi}_{1}(t, j) \in D$ and $\tilde{\phi}_{1}(t, j+1)=$ $g\left(\tilde{\phi}_{1}(t, j)\right), \tilde{\phi}_{2}(t, j) \in C \backslash D$ and $\tilde{\phi}_{2}(t, j+1)=\tilde{\phi}_{2}(t, j)$;

b) if $\tilde{\phi}(t, j) \in(C \backslash D) \times D$, then $\tilde{\phi}_{1}(t, j) \in C \backslash D$ and $\tilde{\phi}_{1}(t, j+1)=$ $\tilde{\phi}_{1}(t, j), \tilde{\phi}_{2}(t, j) \in D$ and $\tilde{\phi}_{2}(t, j+1)=g\left(\tilde{\phi}_{2}(t, j)\right)$; 
c) $\operatorname{if}_{\sim} \tilde{\phi}(t, j) \in D \times D$, then $\tilde{\phi}_{1}(t, j) \in D$ and $\tilde{\phi}_{1}(t, j+1)=g\left(\tilde{\phi}_{1}(t, j)\right)$, $\tilde{\phi}_{2}(t, j) \in D$ and $\tilde{\phi}_{2}(t, j+1)=g\left(\tilde{\phi}_{2}(t, j)\right)$.

We use Algorithm 1 to define a hybrid arc $\phi_{1}$.

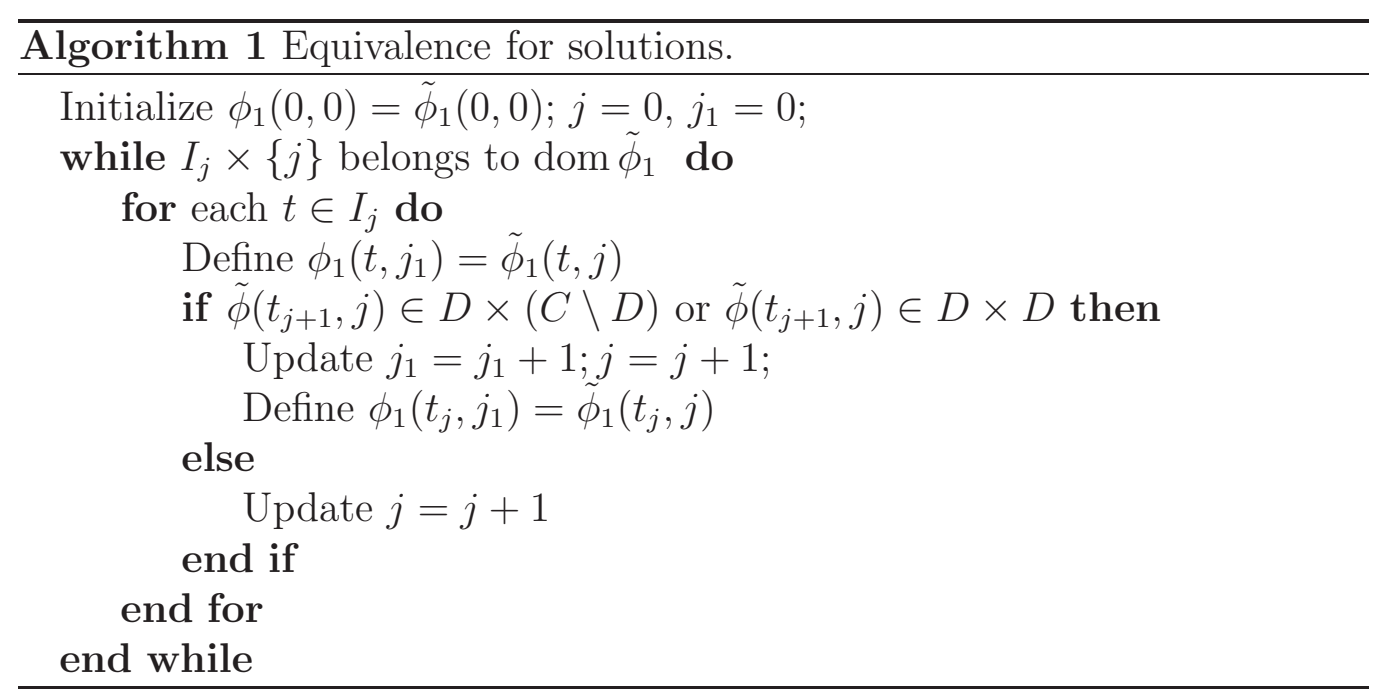

Note that the resulting hybrid arc $\phi_{1}$ mimics the trajectory of $\tilde{\phi}_{1}$ but eliminates the jumps in $\tilde{\phi}_{1}$ that are triggered by the condition $b$ ) above. From this algorithm, for every $(t, j) \in \operatorname{dom} \tilde{\phi}_{1}$, there exists $\left(t, j_{1}\right) \in \operatorname{dom} \phi_{1}$ such that $\phi_{1}\left(t, j_{1}\right)=\tilde{\phi}_{1}(t, j)$. Moreover, by using the property of $\tilde{\phi}, \phi_{1}$ satisfies

- for each $j_{1}$ having a $I_{j_{1}}$ with nonempty interior, $\phi_{1}\left(t, j_{1}\right) \in C$ for each $t \in \operatorname{int} I_{j_{1}}$, and $\dot{\phi}_{1}\left(t, j_{1}\right)=f\left(\phi_{1}\left(t, j_{1}\right)\right)$ for almost all $t \in I_{j_{1}}$,

- for all $\left(t, j_{1}\right) \in \operatorname{dom} \phi_{1}$ such that $\left(t, j_{1}+1\right) \in \operatorname{dom} \phi_{1}$, we have $\phi_{1}\left(t, j_{1}\right) \in$ $D, \phi_{1}\left(t, j_{1}+1\right)=g\left(\phi_{1}\left(t, j_{1}\right)\right)$.

Thus, $\phi_{1}$ is a solution to $\mathcal{H}$ with $\phi_{1}(0,0)=\xi$.

Such a strategy can be applied to $\tilde{\phi}_{2}$ to construct a hybrid arc $\phi_{2}$ such that $\phi_{2}$ is a solution to $\mathcal{H}$ with $\phi_{2}(0,0)=\eta$. Note that the same procedure can be used to construct $\tilde{\phi}$ from $\phi_{1}$ and $\phi_{2}$.

\section{Appendix B. Details of Example 2.5}

The $\delta \mathrm{S}$ property with respect to $x$ is verified as follows. Note that, for the case of $q(0,0)=-1$ and up until the second jump (i.e., $\left(t_{2}, 1\right)$ ), a solution 
from $\phi(0,0)=\left(\phi^{x}(0,0),-1\right) \in C$ is given by $\phi^{x}(t, 0)=-t+\phi^{x}(0,0)$ and $\phi^{q}(t, 0)=-1$ for all $t \in\left[0,1+\phi^{x}(0,0)\right]$, and $\phi^{x}(t, 1)=t-\phi^{x}(0,0)-2$, $\phi^{q}(t, 1)=1$ for all $t \in\left[1+\phi^{x}(0,0), 3+\phi^{x}(0,0)\right]$. Given $\varepsilon>0$, consider any two maximal solutions given by $\phi_{1}=\left(\phi_{1}^{x}, \phi_{1}^{q}\right)$ and $\phi_{2}=\left(\phi_{2}^{x}, \phi_{2}^{q}\right)$ to $\mathcal{H}$ from $\phi_{1}(0,0)$ and $\phi_{2}(0,0)$, such that $\left|\phi_{1}(0,0)-\phi_{2}(0,0)\right| \leq \delta$, where $\delta \in(0, \varepsilon]$ and $\delta$ is small enough to yield $\phi_{1}^{q}(0,0)=\phi_{2}^{q}(0,0)=-1\left(\right.$ or $\phi_{1}^{q}(0,0)=\phi_{2}^{q}(0,0)=1$, which can be proved similarly). Without loss of generality, assume that $\phi_{1}^{x}(0,0)>\phi_{2}^{x}(0,0)$. Then

$$
\begin{aligned}
\left|\phi_{1}^{x}(t, 0)-\phi_{2}^{x}(t, 0)\right| & =\left|-t+\phi_{1}^{x}(0,0)-\left(-t+\phi_{2}^{x}(0,0)\right)\right| \\
& =\left|\phi_{1}^{x}(0,0)-\phi_{2}^{x}(0,0)\right| \leq \delta \leq \varepsilon
\end{aligned}
$$

for $t \in\left[0,1+\phi_{2}^{x}(0,0)\right]$. Since $\phi_{2}$ jumps first, it follows that after the jump $\phi_{2}^{q}\left(1+\phi_{2}^{x}(0,0), 1\right)=1$ and for $t \in\left[1+\phi_{2}^{x}(0,0), 1+\phi_{1}^{x}(0,0)\right]$ we have that

$$
\begin{aligned}
\mid \phi_{1}^{x}(t, 0)- & \phi_{2}^{x}(t, 1)|=|-t+\phi_{1}^{x}(0,0)-\left(t-\phi_{2}^{x}(0,0)-2\right) \mid \\
& \leq\left|-2\left(1+\phi_{2}^{x}(0,0)\right)+2+\phi_{1}^{x}(0,0)+\phi_{2}^{x}(0,0)\right| \\
& \leq\left|\phi_{1}^{x}(0,0)-\phi_{2}^{x}(0,0)\right| \leq \delta \leq \varepsilon .
\end{aligned}
$$

Then, it follows that

$$
\begin{aligned}
\left|\phi_{1}^{x}(t, 1)-\phi_{2}^{x}(t, 1)\right| & =\left|t-\phi_{1}^{x}(0,0)-2-\left(t-\phi_{2}^{x}(0,0)-2\right)\right| \\
& =\left|-\phi_{1}^{x}(0,0)+\phi_{2}^{x}(0,0)\right| \leq \delta \leq \varepsilon
\end{aligned}
$$

for $t \in\left[1+\phi_{1}^{x}(0,0), 3+\phi_{2}^{x}(0,0)\right]$. Proceeding in the same way, we obtain $\left|\phi_{1}^{x}\left(t, j_{1}\right)-\phi_{2}^{x}\left(t, j_{2}\right)\right| \leq \varepsilon$ for each $t>3+\phi_{2}^{x}(0,0)$. Then, the system is $\delta \mathrm{S}$ with respect to $x$.

\section{Appendix C. Details of Example 2.7}

The $\delta$ GAS property of the example can be verified as follows. Note $\mathcal{H}$ also satisfies Assumption 2.1. The arguments for proving incremental attractivity follow similarly to those of Example 2.6.

To prove the $\delta \mathrm{S}$ property, for a given $\varepsilon>0$, pick two solutions $\phi_{1}=$ $\left(\phi_{1}^{x}, \phi_{1}^{q}, \phi_{1}^{\tau}, \phi_{1}^{k}\right)$ and $\phi_{2}=\left(\phi_{2}^{x}, \phi_{2}^{q}, \phi_{2}^{\tau}, \phi_{2}^{k}\right)$ to the system from $\phi_{1}(0,0)$ and $\phi_{2}(0,0)$ such that $\left|\phi_{1}(0,0)-\phi_{2}(0,0)\right|<\delta$, where $\delta$ is chosen such that

$$
\frac{1}{1-\gamma} \delta+(1-\exp (-\delta)) \frac{1+\gamma}{1-\gamma} M \leq \min \{1, \varepsilon\}
$$


Note that $\delta<1$ guarantees that $\phi_{1}^{k}(0,0)=\phi_{2}^{k}(0,0)$. For each $j \in \mathbb{N} \backslash\{0\}$, let $\bar{t}_{j}=\max _{(t, j-1) \in \operatorname{dom} \phi_{1} \cap \operatorname{dom} \phi_{2}} t$ and $\bar{t}_{j}^{\prime}=\min _{(t, j) \in \operatorname{dom} \phi_{1} \cap \operatorname{dom} \phi_{2}} t$. Then, we have that for each $t \in\left[0, \bar{t}_{1}\right]$

$$
\begin{aligned}
\left|\phi_{1}^{x}(t, 0)-\phi_{2}^{x}(t, 0)\right| \leq & \exp (-t)\left|\phi_{1}^{x}(0,0)-\phi_{2}^{x}(0,0)\right| \\
& +(1-\exp (-t))\left|\phi_{1}^{q}(0,0)-\phi_{2}^{q}(0,0)\right| \\
\leq & \exp (-t) \delta+(1-\exp (-t)) \delta \leq \delta \leq \varepsilon,
\end{aligned}
$$

where we used the property $\left|\phi_{1}^{x}(0,0)-\phi_{2}^{x}(0,0)\right| \leq\left|\phi_{1}(0,0)-\phi_{2}(0,0)\right| \leq \delta$ and, likewise, $\left|\phi_{1}^{q}(0,0)-\phi_{2}^{q}(0,0)\right| \leq \delta$. For each $t \in\left[\bar{t}_{1}, \bar{t}_{1}^{\prime}\right]$,

$$
\begin{aligned}
\left|\phi_{1}^{x}(t, 1)-\phi_{2}^{x}(t, 0)\right| \leq & \exp \left(-t+\bar{t}_{1}\right)\left|\left(\phi_{1}^{x}\left(t_{1}, 0\right)-\phi_{2}^{x}\left(\bar{t}_{1}, 0\right)\right)\right| \\
& +\left(1-\exp \left(-t+\bar{t}_{1}\right)\right)\left|\gamma \phi_{1}^{q}(0,0)+\phi_{2}^{q}(0,0)\right| \\
\leq & \delta+(1-\exp (-\delta))(1+\gamma) M \leq \varepsilon,
\end{aligned}
$$

where we used the fact that $\phi_{1}^{q}(0,0), \phi_{2}^{q}(0,0) \in[-M, M]$ and (C.1). In fact, for each $t \in\left[\bar{t}_{i-1}^{\prime}, \bar{t}_{i}\right]$, where $i \in \mathbb{N} \backslash\{0,1\}$,

$$
\begin{aligned}
\left|\phi_{1}^{x}(t, i-1)-\phi_{2}^{x}(t, i-1)\right| \leq & \exp \left(-t+\vec{t}_{i-1}^{\prime}\right)\left|\left(\phi_{1}^{x}\left(\vec{t}_{i-1}^{\prime}, i-1\right)-\phi_{2}^{x}\left(\vec{t}_{i-1}^{\prime}, i-1\right)\right)\right| \\
& +\left(1-\exp \left(-t+\vec{t}_{i-1}^{\prime}\right)\right) \gamma^{i-1}\left|\phi_{1}^{q}(0,0)-\phi_{2}^{q}(0,0)\right| \\
\leq & \sum_{k=0}^{i-1} \gamma^{k} \delta+(1-\exp (-\delta)) \sum_{k=0}^{i-2} \gamma^{k}(1+\gamma) M \leq \varepsilon
\end{aligned}
$$

Moreover, for each $t \in\left[\bar{t}_{i}, \bar{t}_{i}^{\prime}\right]$, where $i \in \mathbb{N} \backslash\{0,1\}$,

$$
\begin{aligned}
\left|\phi_{1}^{x}(t, i)-\phi_{2}^{x}(t, i-1)\right| \leq & \exp \left(-t+\bar{t}_{i}\right)\left|\left(\phi_{1}^{x}\left(\bar{t}_{i}, i-1\right)-\phi_{2}^{x}\left(\bar{t}_{i}, i-1\right)\right)\right| \\
& +\left(1-\exp \left(-t+\bar{t}_{i}\right)\right) \gamma^{i-1}\left|\gamma \phi_{1}^{q}(0,0)+\phi_{2}^{q}(0,0)\right| \\
\leq & \sum_{k=0}^{i-1} \gamma^{k} \delta+(1-\exp (-\delta)) \sum_{k=0}^{i-1} \gamma^{k}(1+\gamma) M \leq \varepsilon .
\end{aligned}
$$

Therefore, the system is $\delta \mathrm{S}$ with respect to $x$ leading to the fact that $\mathcal{H}$ is $\delta \mathrm{GAS}$ with respect to $x$. 